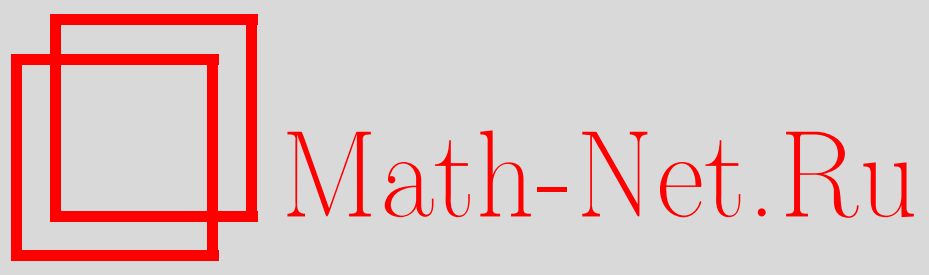

А. Г. Мясников, В. Н. Ремесленников, Е. В. Френкель, Свободное произведение групп с объединением: нормальные формы и меры, Матем. заметки, 2012, том 91, выпуск 4, 633-637

DOI: https://doi.org/10.4213/mzm9328

Использование Общероссийского математического портала Math-Net.Ru подразумевает, что вы прочитали и согласны с пользовательским соглашением http://www . mathnet.ru/rus/agreement

Параметры загрузки:

IP : 54.224 .187 .69

26 апреля 2023 г., $17: 23: 21$

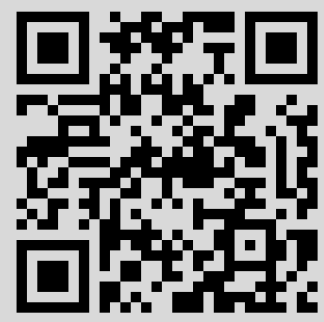




\section{Свободное произведение групп с объединением: нормальные формы и меры}

\section{А. Г. Мясников, В. Н. Ремесленников, Е. В. Френкель}

1. Введение и формулировка основных результатов. Пусть $G=A_{C}^{*} B$ - свободное произведение с объединением и $A, B, C$-свободные группы конечных рангов. В этом классе групп существуют группы, достаточно сложные с алгоритмической точки зрения: например, группы с неразрешимой проблемой сопряженности [1], [2]. Следовательно, если мы понимаем сложность алгоритма с классической точки зрения, то сложность алгоритма для решения проблемы сопряженности на множителях $A$ и $B$ значительно отличается от ее сложности на группе $G$. Однако, согласно исследованиям последних лет [3], [4] размер тех входных данных алгоритмов на группе $G$, для которых проблема сопряженности решается сложно, либо алгоритм вообще не дает ответа, пренебрежим в некотором точном математическом смысле по сравнению с размером всех входов. Вот один из примеров такого сорта результатов.

Tеорема 1 [3; следствие 4.19]. Пусть $G=A \underset{C}{*} B$ - свободное произведение с объединением и $A, B, C$ - свободные группы конечных рангов. Тогда поисковая проблема сопряженности в $G$ разрешима для всех сопряженных к ииклически редуцированным регулярным элементам группы $G$.

В связи с этим и другими результатами подобного рода возникают следующие проблемы: во-первых, нужно уметь оценивать в подходящей мере множество элементов группы $G$, записанных в нормальной форме, а также оценивать плотность в них подмножеств регулярных нормальных форм; во-вторых, показать, что на множестве регулярных нормальных форм проблема сопряженности решается за полиномиальное время.

Решению первой задачи посвящены работы [4], [5] и данная статья, в которой в конспективной форме представлены основные результаты (теоремы А, В) развернутой статьи [6]. Начнем с формулировки основных теорем. Необходимые пояснения к теоремам будут приведены позже.

Теорема А. Пусть $G=\underset{C}{*} B-$ свободное произведение групп с объединением и $A, B$, $C$ - свободные группы конечных рангов. Тогда

(i) если $C$ имеет конечный индекс в $A$ и $B$, то всякая нормальная форма сингулярна и нестабильна;

(ii) если $C$ бесконечного индекса хотл бы в одной из групп $A$ или $B$, mо $\mathscr{N}_{r}$ и $\mathscr{N F}_{s}$ экспоненииально $\mu$-генерические относительно $\mathscr{N} \mathscr{F}$, a $\mathscr{N} \mathscr{F}_{\sin } u \mathscr{N} \mathscr{F}$ uns экспоненицально $\mu$-пренебрежимые относительно $\mathscr{N} \mathscr{F}$ в следующих случаях:

(ii.1) $\mu$ определяется по псевдомерам $\mu_{A} u \mu_{B}$, которые являются функциями мощности на $A$ и $B$ соответственно, а $\rho_{\mu}$ - двумерная асимптотическая плотность;

(ii.2) $\mu$ определяется по атомарным вероятностным мерам $\mu_{A, l}$ u $\mu_{B, l}$ на $A$ и $B$ соответственно, а $\rho_{\mu_{l}}$ - двумерная асимптотическая плотность по Чезаро.

Теорема В. Пусть $G=A_{C}^{*} B-$ свободное произведение групп с объединением и $A$, $B, C$ - свободные группы конечных рангов. Если $C$ имеет бесконечный индекс хотя бы

Работа выполнена при поддержке Российского фонда фундаментальных исследований (грант № 08-01-00067-a). 
в одной из групп $A$ или $B$, то множества всех нестабилъных $\mathscr{N} \mathscr{F}$ uns $и$ всех сингулярных

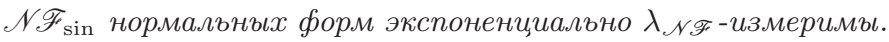

Теперь мы приведем основные определения и результаты, необходимые для понимания теорем А и В.

2. Измерение подмножеств свободной группы. Пусть $F=F(X)$ - свободная группа конечного ранга. Обозначим через $S_{n}$ сферу радиуса $n$ в $F$. Пусть $\mu$ будет атомарной псевдомерой на $F$. Напомним, что мера $\mu$ на счетном множестве $P$ называется атомарной, если каждое подмножество $Q \subseteq P$ измеримо; при этом также выполняется $\mu(Q)=\sum_{q \in Q} \mu(q)$.

Для множества $R \subseteq F$ определим его сферическую асимптотическую плотность относительно меры $\mu$ как следующий предел:

$$
\rho_{\mu}(R)=\varlimsup_{n \rightarrow \infty} f_{\mu, n}(R), \quad \text { где } \quad f_{\mu, n}(R)=\frac{\mu\left(R \cap S_{n}\right)}{\mu\left(S_{n}\right)} .
$$

Далее, пусть $\mu$ - псевдомера на $F$ и $\rho_{\mu}(R)$ - сферическая асимптотическая плотность. Множество $R \subseteq F$ называется генерическим относительно меры $\mu$, если $\lim _{n \rightarrow \infty} f_{\mu, n}(R)$ существует и $\rho_{\mu}(R)=1$, и пренебрежимым относительно меры $\mu$, если $\rho_{\mu}(R)=0$. Далее, будем говорить, что $R$ является экспоненииально генерическим относительно $\mu$, если существует положительное число $\delta<1$ такое, что $1-\delta^{n}<f_{\mu, n}(R)<1$ для достаточно больших $n$. Если же $f_{\mu, n}(R)<\delta^{n}$ для всех достаточно больших $n$, то $R$ называется экспоненииально пренебрежимым относительно меры $\mu$. Далее мы будем опускать слова "относительно меры $\mu$ ", если в качестве $\mu$ берется мощность множества.

3. Стратификация и измерение шрайеровых систем представителей в свободной группе. Пусть $C$ - конечно порожденная подгруппа свободной группы $F=F(X)$. Рассмотрим множество $S$ представителей правых классов смежности группы $F$ по $C$. Система представителей $S$ называется шрайеровой, если всякий начальный сегмент элемента из $S$ тоже лежит в $S$. Представитель $s \in S$ назовем сингулярным, если он приналежит обобщенному нормализатору $C$ :

$$
N_{F}^{*}(C)=\left\{f \in F \mid f^{-1} C f \cap C \neq 1\right\} ;
$$

обозначим множество всех таких представителей через $S_{\sin }$. Все прочие представители назовем регулярными. Представитель $s \in S$ назовем стабилъным, если $s c \in S$ для любого $c \in C$. Обозначим через $S_{\text {uns }}$ множество всех нестабилъных представителей в $S$. В [4] (следствие 5.12) было доказано следующее

УтверЖдениЕ 1. Пусть $C$ - конечно порожденная подгруппа бесконечного индекса в $F(X)$ и $S$ - шрайерова трансверсаль для $C$. Тогда множества сингулярных представителей $S_{\mathrm{sin}}$ и нестабильных представителей $S_{\mathrm{uns}}$ экспоненииально пренебрежимы относительно $S$.

4. Нормальные формы. Приведем четыре основных метода представления элементов группы $G$ в нормальной форме (в п. 3) содержится два метода).

1) В свободно-редуцированной форме: $g=g_{1} g_{2} \ldots g_{n}$, где $g_{i}$ - редуцированные слова в алфавите $X \cup X^{-1}$ или $Y \cup Y^{-1}$, и если $g_{i} \in A$, то $g_{i+1} \in B$, и наоборот. Обозначим $\mathscr{E} \mathscr{F}$ множество всех элементов в свободно-редуцированной форме.

2) В редуцированной форме: $g=c g_{1} g_{2} \ldots g_{k}$, где $c \in C, g_{i} \in(A \cup B) \backslash C$, и $g_{i}$ с $g_{i+1}$ принадлежат различным множителям. Обозначим множество всех элементов в редуцированной форме через $\mathscr{R} \mathscr{F}$. 
3) В единственной (см. [7]) канонической нормальной форме: $g=c p_{1} p_{2} \ldots p_{l}$, где $c \in C$, $p_{i} \in(S \cup T) \backslash\{1\}$, и $p_{i}$ и $p_{i+1}$ принадлежат различным множителям; пусть $\mathscr{C} \mathscr{N} \mathscr{F}$ означает множество всех элементов в такой форме. Определение циклически редуцированной формы см. в [7]; множество всех таких форм обозначим $\mathscr{C} \mathscr{R} \mathscr{F}$.

Будем говорить, что элемент записан в нормальной форме, если он лежит в одном из множеств $\mathscr{E} \mathscr{F}, \mathscr{R} \mathscr{F}, \mathscr{C} \mathscr{N} \mathscr{F}, \mathscr{C} \mathscr{R} \mathscr{F} ;$ далее $\mathscr{N} \mathscr{F}=\{\mathscr{E} \mathscr{F}, \mathscr{R} \mathscr{F}, \mathscr{C} \mathscr{N} \mathscr{F}, \mathscr{C} \mathscr{R} \mathscr{F}\}$. Элемент $g \in$ $F(X)$ мы называем регулярным (стабилъным, и т.д.), если он может быть представим в форме $g=c s, c \in C, s \in S$ и $s$ регулярен, стабилен, и т.д. Элемент $g \in \mathscr{N} \mathscr{F}$ называется регулярным (стабилъным), если по крайней мере один из элементов $g_{i}$ или $p_{i}$ регулярен (стабилен). В противном случае $g$ называется сингулярным (нестабилъным).

Из результатов работы [3] следует, что стабильные и регулярные формы реализуют идею компоненты, на которой алгоритм работает хорошо, тогда как дополняющие множества, наоборот, являют собою "плохую" компоненту в множестве всех нормальных форм. Обозначим через $\mathscr{N F}_{r}\left(\mathscr{N}_{S}\right)$ множество всех регулярных (стабильных) элементов в нормальной форме и через $\mathscr{N} \mathscr{F}_{\sin }(\mathscr{N} \mathscr{F}$ uns $)$ - его дополнение в $\mathscr{N} \mathscr{F}$.

5. Асимптотические плотности свободных произведений множеств. Пусть $\mu_{A}$ и $\mu_{B}$ - атомарные псевдомеры на $A$ и $B$ соответственно и $\mu_{A}(1)=\mu_{B}(1)$. Зафиксируем вероятностное распределение $\theta: \mathbb{N} \rightarrow \mathbb{R}^{+}$; в частности, $\sum_{k=1}^{\infty} \theta(k)=1$. Определим атомарную вероятностную меру $\mu$ на $F=A * B$ как

$$
\mu(f)=\frac{1}{2} \theta(k) \mu_{F_{1}}\left(f_{1}\right) \cdots \mu_{F_{k}}\left(f_{k}\right),
$$

где $f \in \mathscr{E} \mathscr{F}$. Тогда для множества $R \subseteq F$ по определению $\mu(R)=\sum_{f \in R} \mu(f)$. Если $\mu_{A}, \mu_{B}-$ атомарные вероятностные меры на $A$ и $B$ соответственно, то $\mu$-атомарная вероятностная мера на $F$.

Далее, пусть $T=A_{0} * B_{0} \subseteq F=A * B$. Для пары натуральных чисел $(n, k)$ определим

$$
T_{n, k}=\left\{f=f_{1} \ldots f_{k} \in T: s(f)=k,\left|f_{i}\right| \leqslant n, i=1, \ldots, k\right\} .
$$

Тогда $T=\bigcup_{k=0, n=0}^{\infty} T_{n, k}$. Для множества $Q=A_{1} * B_{1}$ в $F$ положим

$$
\rho_{\mu}^{n, k}(Q, T)=\frac{\mu\left(Q \cap T_{n, k}\right)}{\mu\left(T_{n, k}\right)}
$$

Асимптотическое поведение $Q$ относительно $T$ мы будем характеризовать двумерной асимптотической плотностью, которую определим как следующий предел:

$$
\rho_{\mu}(Q, T)=\varlimsup_{d(n, k) \rightarrow \infty} \rho_{\mu}^{n, k}(Q, T),
$$

где под функцией направления $d(n, k)$ мы понимаем некоторый взаимнооднозначный между $n$ и $k$ путь из $(1,1)$ в $(\infty, \infty)$. Если указанный предел существует и не зависит от выбора направления, обозначим его через $\rho_{\mu}^{\mathrm{e}}(Q, T)$. Множество $Q$ является $\mu$-генерическим относительно $T$, если $\rho_{\mu}^{\mathrm{e}}(Q, T)=1$, и $\mu$-пренебрежимым относительно $T$, если $\rho_{\mu}^{\mathrm{e}}(Q, T)=0$. Назовем $Q$ экспоненциально $\mu$-генерическим относительно $T$, если $Q$ является $\mu$-генерическим и существует положительная константа $\delta<1$ такая, что, начиная с некоторого $k$, выполняется $1-\delta^{k}<\rho_{\mu}^{n, k}(Q, T)<1$. Если $Q$ является $\mu$-пренебрежимым и $\rho_{\mu}^{n, k}(Q, T)<\delta^{k}$ для достаточно больших $k$, то $Q$ называется экспоненциально $\mu$-пренебрежимым относительно $T$. 
6. Атомарные меры элементов в нормальной форме. Вероятность получить элемент $g \in \mathscr{E} \mathscr{F}(g \in \mathscr{R} \mathscr{F})$ слоговой длины $k$ равна

$$
\mu_{k}(g)=\frac{1}{2} \mu_{1}\left(g_{1}\right) \cdots \mu_{k}\left(g_{k}\right)
$$

где $\mu_{i}\left(g_{i}\right)=\mu_{A}\left(g_{i}\right)$, если $g_{i} \in A$, и $\mu_{i}\left(g_{i}\right)=\mu_{B}\left(g_{i}\right)$ в противном случае, $i=1, \ldots, k, k \geqslant 1$; если $k=0$, то $\mu_{0}(c)=\mu_{C}(c)$, где $\mu_{C}(c)=\mu_{A, C}$, если $C$ рассматривается как подгруппа $A$, и $\mu_{C}(c)=\mu_{B, C}$ иначе.

Вероятностная мера на элементах $g \in \mathscr{C} \mathscr{N} \mathscr{F}(g \in \mathscr{C} \mathscr{R} \mathscr{F})$ слоговой длины $k$ равна

$$
\mu_{k}(g)=\frac{1}{2} \mu_{C}(c) \mu_{1}\left(p_{1}\right) \mu_{2}\left(p_{2}\right) \cdots \mu_{k}\left(p_{k}\right),
$$

где $\mu_{i}\left(p_{i}\right)=\mu_{F\left(p_{i}\right)}\left(C p_{i}\right), i=0, \ldots, k$, и $\mu_{C}(c)$ определена выше.

Вероятностные меры $\mu_{A}, \mu_{B}$ и $\mu_{C}(c)$ и др. могут быть определены, как и в [5], [8] и [4] при помощи невозвратного случайного блуждания на некотором графе для $A, B$ или их подгруппы $C$.

Вероятностная мера $\mu$ на множестве $\mathscr{N} \mathscr{F}$ по определению равна $\mu(g)=\theta(k) \mu_{k}(g)$.

7. Меры на множествах нормальных форм: два подхода. В п. 2 мы определили асимптотические плотности подмножеств свободной группы внутри самой группы $F=$ $F(X)$; здесь же, следуя [4], мы введем понятие асимптотической плотности множества $R \subseteq F$ относительно другого множества $L \subseteq F$. Пусть

$$
f_{n}(R, L)=\frac{\left|R \cap S_{n}\right|}{\left|L \cap S_{n}\right|} .
$$

Aсимптотическая плотность $\rho(R, L)$ множества $R$ относительно $L$ вычисляется как $\rho(R, L)=\varlimsup_{n \rightarrow \infty} f_{n}(R, L)$. Понятия (экспоненциально) пренебрежимого и генерического множества относительно $L$ могут быть введены так же, как и в п. 2.

В [8] были также введены понятия $\lambda_{L}$-измеримого и экспоненциально $\lambda_{L}$-измеримого множества $R$. Именно,

$$
\lambda_{L}(R)=\sum_{w \in R} \lambda_{L}(w)=\sum_{n=0}^{\infty} f_{n}^{\prime}(R, L),
$$

где

$$
f_{n}^{\prime}(R, L)=\sum_{w \in R \cap S_{n}} \lambda_{L}(w)
$$

и $\lambda_{L}(w), f_{n}^{\prime}(R, L)$ определяются случайным блужданием на некотором автомате для $L$ (см. более подробно в [4; п. 5.2 , с. 109]). Отметим, что вообще говоря, частоты $f_{k}^{\prime}(R, L)$ отличаются от относительных частот $f_{k}(R, L)$, определенных выше, в силу специфики автомата для $L$. С другой стороны, эти частоты совпадают для $L=F(X)$, т.е. $f_{n}^{\prime}(R, F(X))=$ $f_{n}(R, F(X))$.

Множество $R$ назовем $\lambda_{L}$-измеримым, если $\lambda_{L}(R)$ конечно. Множество $R$ будем называть экспоненциально $\lambda_{L}$-измеримым, если $f_{n}^{\prime}(R, L) \leqslant \delta^{n}$ для всех достаточно больших $n$ и положительной константы $\delta<1$.

Для всякого $w \in F$ множество $C_{L}(w)=L \cap C(w)$ называется $L$-конусом, и конус $C_{L}(w)$ называется $L$-малым, если он экспоненциально $\lambda_{L}$-измерим; в противном случае конус называется большим. В работе [4] был также доказан следующий результат.

Теорема 2 [5; теорема 5.4]. Пусть $R$-регулярное подмножество префиксно-замкнутого регулярного множества $L$ в свободной группе $F$ конечного ранга. Тогда либо префиксное замыкание $\bar{R}$ множества $R$ в $L$ содержит большой $L$-конус, либо $\bar{R}$ экспоненииально $\lambda_{L}$-измеримо.

Эту теорему мы применяем при доказательстве теоремы В. 


\section{СПИСОК ЦИТИРОВАННОЙ ЛИТЕРАТУРЫ}

[1] С. И. Адян, В. Г. Дурнев, УМН, 55:2 (2000), 3-94. [2] С. F. Miller III, Algorithms and Classification in Combinatorial Group Theory, Math. Sci. Res. Inst. Publ., 23, Springer, New York, 1992, 1-59. [3] A. V. Borovik, A. G. Myasnikov, V. N. Remeslennikov, Internat. J. Algebra Comput., 17:7 (2007), 1299-1333. [4] E. Frenkel, A. G. Myasnikov, V. N. Remeslennikov, Combinatorial and Geometric Group Theory, Trends Math., Birkhäuser Verlag, Basel, 2010, 93-118. [5] A. V. Borovik, A. G. Myasnikov, V. N. Remeslennikov, Internat. J. Algebra Comput., 13:6 (2003), 705-731. [6] А. Г. Мясников, В.Н.Ремесленников, Е. В. Френкель, Фундамент. и прикл. матем., 16:8 (2010), 189-221. [7] В. Магнус, А. Каррасс, Д. Солитер, Комбинаторная теория групп. Представление групп в терминах образующих и соотношений, Наука, M., 1974. [8] W. Woess, Arch. Math. (Basel), 41:4 (1983), 363-370.

\section{А. Г. Мясников}

Charles V. Schaefer, Jr. School of Engineering \&

Science, Stevens Institute of Technology, USA

E-mail: amiasnik@stevens.edu

\section{В. Н. Ремесленников}

Омский филиал Института математики

им. С. Л. Соболева СО РАН

\section{Е. В. Френкель}

Московский государственный университет им. М. В. Ломоносова

E-mail: lizzy.frenkel@gmail.com 